\title{
Évolution du système immunitaire : acquisition de la diversité des anticorps ${ }^{(1)}$
}

Le système immunitaire des vertébrés évolue depuis 600 millions d'années, lorsque sont apparus, chez les poissons primitifs, l'essentiel des gènes et cellules spécifiques de ce système. Les solutions choisies au cours de la phylogenèse pour engendrer la diversité des anticorps (et des récepteurs des lymphocytes $T$ ) constituent une variation sur un thème, faisant de façon variable appel au réarrangement combinatoire d'éléments génétiques, à la conversion génique et aux mutations somatiques. Par certains aspects, les systèmes sélectionnés reflètent plus une bonne adaptation aux caractéristiques et à la dynamique des éléments cellulaires du système immunitaire (durée de la période utilisable pour le développement du répertoire, nombre de cellules spécialisées disponibles, activité mitotique de ces cellules, etc.) que la filiation entre les espèces.

\section{Louis Du Pasquier}

\section{ADRESSE}

L. Du Pasquier : docteur ès sciences, membre permanent du Basel Institute for Immunology Ehrendozent à l'université de Bâle. Basel Institute for Immunology*, 487, Grenzacherstrasse, CH-4058, Bâle, Suisse.

(1) Cet article a fait l'objet d'une présentation orale à la 4 e Journée Nationale d'Actualités en Immunologie, organisée par les Laboratoires Cassenne, à Paris, le 15 juin 1991

\footnotetext{
* Fondé et financé par Hoffmann-La Roche. $\mathrm{m} / \mathrm{s} n^{\circ} 7$, vol. 7 , septembre 91
}

e système immunitaire offre plusieurs particularités qui rendent son étude phylogénique très originale. (1) Complexe assemblage de gènes apparentés, il est un exemple frappant d'évolution par duplication. (2) L'état d'équilibre dans lequel nous le trouvons chez les différentes espèces à l'heure actuelle nous le montre pourvu de capacités évolutives rapides liées à l'existence d'un grand nombre de gènes semblables et homologues favorisant les crossing-over inégaux. Il faut donc s'attendre à une flexibilité du système et à des variations importantes dans le nombre et la position de ses loci génétiques d'une espèce à l'autre, surtout si l'on tient compte des adaptations diverses auxquelles les composants du système immunitaire sont confrontés. (3) Le système imunitaire forme un tout dont l'évolution indépendante d'une partie peut engendrer des modifications profondes dans les autres éléments: l'ensemble des gènes du complexe majeur d'histocompatibilité, des immunoglobulines, du récepteur des cellules T, n'évoluera pas de la même manière selon la façon dont les cellules $B$ et $T$ seront produites. Étudier l'évolution du système immunitaire c'est (peutêtre plus que dans d'autres systèmes physiologiques) étudier la coévolution de plusieurs systèmes. (4) Le système immunitaire est l'un des rares systèmes physiologiques qui évoluent somatiquement au cours de l'ontogenèse selon les mêmes principes que l'évolution phylogénique, c'est-à-dire par mutations et sélection. Cela est vrai pour la genèse des cellules $B$ et $T$ chez tous les vertébrés étudiés jusqu'à présent, même 
si les modalités varient quelque peu d'une espèce à l'autre. (5) Le système immunitaire des vertébrés semble être apparu brusquement chez les poissons primitifs avec l'essentiel de son cortège de gènes, de molécules et de cellules. Le problème de son origine en est d'autant plus compliqué. La plupart de ses éléments semblent être présents même dans les systèmes les plus simples. Cela n'est peut-être que le résultat d'une standardisation par sélection, comme c'est souvent le cas au cours de l'évolution. Il n'est pas impossible qu'un grand nombre d'expériences évolutives aient été tentées chez les premiers vertébrés, dont les représentants ont disparu. Son évolution ultérieure à partir d'éléments conservés obéit peut-être à un programme " égoïste " interne lié aux propriétés des gènes. Étant donné ces restrictions, on n'observera peut-être, chez les vertébrés, que des variations sur un même thème. Quant à l'origine du système immunitaire, l'étude des invertébrés révèle de nombreux cas d'homologies et d'analogies structurelles suggérant une origine très ancienne du système immunitaire, ou tout au moins de bon nombre de ses éléments.

7. Du Pasquier L. The genetic control of histocompatibility reactions ; phylogenetic aspects. Arch Biol 1974; 85 : 91-103.

8. Valembois P, Roch P, Boiledieu D. Cel lular defence systems of the Platyhelminthes, Nemertea, Sipunculida and Annelida, In Cohen N, Sigel M, eds The ReticuloEndothelial System Vol. 3, New York: Plenum, 1983 : 89-139.

9. Baynes CJ. Phagocytosis and nonself recognition in invertebrates. Bioscience 1990 . $10: 723-31$.

10. Seeger MA, Haffley L, Kaufman TC. Characterization of amalgam, a member of the Ig superfamily from Drosophila. Cell 1988 ; 55 : 589-600.

11. Harding FA., Amemiya CT, Litman RT, Cohen N, Litman GW. Two distinct immunoglobulin heavy chain isotypes in a primitive, cartilaginous fish, Raja erinacea, Nucleic Acids Res 1990 ; 18 : 6369-76.

12. Schwager J, Mikoryak CA, Steiner LA. Aminoacid sequence of heavy chains from Xenopus laevis IgM deduced from cDNA sequences: implication for evolution of immunoglobulin domains. Proc Natl Acad Sci USA $1988 ; 85$ : 2245-9.

13. Schwager J, Burckert N, Schwager M, Wilso NM. Evolution of immunoglobulin light chain genes : analysis of Xenopus Ig L isotypes and their contribution to antibody diversity. EMBO J $1991 ; 10: 505-11$.

\section{Origine du système immunitaire des vertébrés}

Le règne animal est constitué d'environ 20 embranchements dans lesquels on peut rechercher les paramètres du système immunitaire (figure 1) [1].

- Reconnaissance des polymorphismes allogéniques et son contrôle génétique. Dans de nombreux embranchements, les expériences de transplantation allogénique (c'est-àdire entre individus de la même espèce) révèlent l'existence de mécanismes précis de distinction entre le soi et le non-soi. Ceux-ci sont souvent sous la dépendance d'une région génétique unique ou majeure (cas des porifères, cnidaires, tuniciers). L'analogie est frappante avec le complexe majeur d'histocompatibilité des vertébrés supérieurs, mais, alors que chez ces derniers les réactions allogéniques semblent ne constituer qu'une manifestation accessoire et très peu naturelle d'autres fonctions, il n'en n'est pas de même chez de nom-

breux invertébrés marins. En effet, les fonds aquatiques sont souvent le théâtre d'une compétition pour l'espace. Des contacts fréquents se produisent entre individus d'une même espèce en cours de croissance. La reconnaissance allogénique est une condition sine qua non de survie en tant qu'individu, alors qu'elle a perdu cette fonction chez les vertébrés [2]. Le mode de vie de nombreux invertébrés peut donc constituer une base de sélection pour ces mécanismes de reconnaissance. On a aussi proposé que ces mécanismes éviteraient le parasitage d'une espèce par les cellules germinales d'une autre [3]. Il est possible que les vertébrés aient tiré parti de tels mécanismes, après les avoir intériorisés et modifiés au profit des interactions entre les différents types cellulaires du système immunitaire. Certains auteurs préfèrent rechercher les origines du système immunitaire à l'intérieur de chaque individu et pensent qu'il pourrait s'être développé à partir des systèmes de reconnaissance de surface engagés dans les interactions cellulaires présidant à la genèse des tissus et des organes des métazoaires [4, 5]. N'oublions pas, cependant, que l'on rencontre des phénomènes de reconnaissance allogénique chez des protozoaires.

- Mémoire immunologique. La distinction entre le soi et le non-soi peut en principe se faire de plusieurs manières. (1) On peut, par référence à soi-même, distinguer en bloc tout ce qui est étranger. En d'autres termes, on rejettera l'autre s'il ne porte pas les marqueurs du soi, mais on ne se préoccupe pas de la nature de ses marqueurs. (2) On peut aussi reconnaître spécifiquement les différents non-soi à l'aide d'une batterie de structures de reconnaissance spécifiques. Ce mode spécifique entraîne la possibilité de réponses mesurées, de mémoire spécifique (donc de réactions plus rapides), de la constitution de clones, et donc d'une plus grande finesse dans les réactions suivant le phénomène de reconnaissance à proprement parler. En conséquence, la présence ou l'absence d'une mémoire spécifique nous renseigne sur le mode de reconnaissance pratiqué par les 


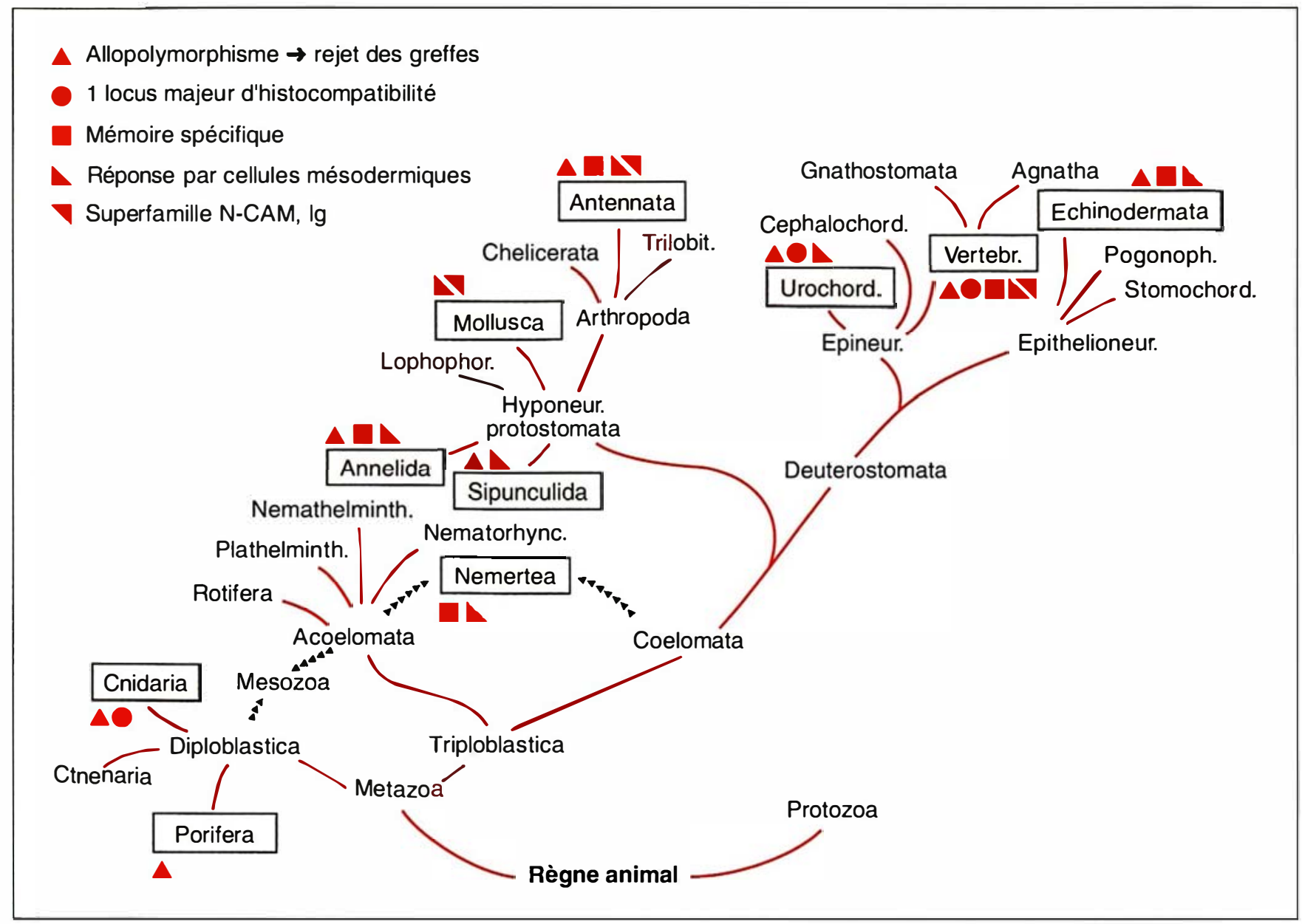

Figure 1. Les éléments du système immunitaire dans le règne animal. (Emprunté et traduit de la référence [1].)

espèces. La figure 1 nous montre que cette mémoire est présente de façon non équivoque chez tous les métazoaires de type triploblastique (références pour cette question dans [6]). On peut donc conclure que tous ces embranchements ont sélectionné un mode de reconnaissance spécifique, ce qui ne veut pas dire que ces systèmes soient nécessairement apparentés entre eux. On peut assister à un phénomène de convergence. Il est plus difficile d'obtenir des renseignements sur le mode de reconnaissance des espèces ne montrant pas de mémoire immunologique spécifique. Chez ces derniers, c'est le mode de contrôle génétique de réactions d'histocompatibilité qui constitue une source d'information. Si une seule différence allélique entraîne une réaction de rejet, on peut conclure à l'existence d'un mécanisme de reconnaissance des différents non-soi. En effet, cela $m / s n^{\circ} 7$, col. 7 , septembre 91 signifie que, dans ce cas, la reconnaissance de l'allèle commun n'a pas suffi à entraîner la compatibilité et que la différence a été reconnue pour elle-même. Les expériences de transplantation effectuées dans des familles de métazoaires primitifs (les cnidaires, animaux diploblastiques) mais aussi chez les tuniciers, invertébrés beaucoup plus avancés dans leur organisation et que l'on considère comme assez proches des vertébrés, montrent qu'en général une seule différence allélique au locus majeur d'histocompatibilité n'entraîne pas de manifestations visibles de rejet. Certains en ont conclu que les invertébrés ne possédaient pas de mécanismes de reconnaissance spécifique des différents non-soi. Cependant, un tel mode de réactivité peut simplement refléter un effet de seuil. Ainsi les amphibiens - qui, à l'état adulte, reconnaissent efficacement une scule différence allélique au niveau du complexe majeur d'histocompatibilité - ne le font pas au moment de la métamorphose, sans pour autant que leur mode de reconnaissance ait changé [7]. Des études fines d'histologie chez les tuniciers ont effectivement montré que, dans les cas de transplantation entre individus différant par un seul allèle du locus d'histocompatibilité, on pouvait trouver des signes cytologiques de réaction, et non pas de tolérance. On peut donc penser que, dans l'ensemble du règne animal, la reconnaissance allogénique s'effectue selon les mêmes modalités.

- Origine mésodermique des cellules effectrices de la mémoire immunologique. On remarque sur la figure 1 une corrélation frappante entre l'existence d'une mémoire immunologique et l'existence d'un 


\section{RÉFÉRENCES}

14. Zezza DJ, Mikoryak CA, Schwager J, Steiner LA. Sequence of constant region of light chains from Xenopus laevis immunoglobulins. J Immunol 1991 (sous presse).

15. Kokubu F, Hinds K, Litman R, Shamblott MS, Litman GW. Complete structure and orgazination of immunoglobulin heavy chain constant region genes in a phylogenetically primitive vertebrate. $E M B O \mathrm{~J}$ $1978 ; 7$ : 1979-88

16. Litman GW, Amemiya CT, Haire RN, Shamblott MJ. Antibody and immunoglobulin diversity. Bioscience $1990 ; 40: 751-7$

17. Du Pasquier L, Schwager J, Flajnik MF. The immune system of Xenopus. Ann Rev Immunol $1989 ; 7$ : 251-75.

18. Reynaud CA, Anquez V, Dahan A Weill JC. A single rearrangement event generates most of the chicken immunoglobulin light chain diversity. Cell 1985 ; 40 : 283-91.

19. Becker RS, Knight KL. Somatic diversification of Ig heavy chain and VDJ genes : evidence for somatic gene conversion in rabbits. Cell $1990 ; 63$ : 987-97.

20. Du Pasquier L. Antibody diversity in lower vertebrates. Why it it so restricted? Nature 1982 ; 296 : 311-3

21. Alt FW, Blackwell TK, Yancopoulos GD. Development of the primary antibody repertoire. Science 1987 ; 238 : 1079-87.

22. Du Pasquier L, Schwager J. Immunoglobulin genes and B cell development in amphibians. In : Gupta S, Cooper MD Paul W, eds Proc. Int. Conf. on Lymphocyte Physiology. 1991 (sous presse).

23. Weill JC, Reynaud CA. The chicken B cell compartment. Science 1987; 238 : 1094-8.

24. Reynaud CA, Mackay CR, Müller RG, Weill JC. Somatic generation of diversity in a mammalian primary lymphoid organ. The sheep ileal Peyer's patches. Cell $1991 ; 64$ : 995-1005

25. Kobel HR, Du Pasquier L. Genetics of polyploid Xenopus. Trends Genet 1986 ; 2 : 310-5.

26. Du Pasquier L, Hsu E. Immunoglobulin expression in diploid and polyploid interspecies hybrids of Xenopus : evidence for allelic exclusion. Eur J Immunol $1983 ; 13$ : 585-90.

27. Ghaffari SH, Lobb CJ. Heavy chain variable region gene families evolved early in phylogeny. I Immunol 1991; 146 mésoderme et plus précisément d'un coelome. Ce feuillet est à l'origine des cellules du sang notamment des leucocytes circulants. Chez de nombreux invertébrés, ces cellules se développent à partir de la splanchnopleure entourant le tube digestif [8]. Il en est de même pour les lymphocytes des vertébrés supérieurs, qui se différencient et prolifèrent dans des organes associés au tube digestif, faisant intervenir des associations entre tissus dérivés du mésoderme et tissus dérivés de l'endoderme, comme le thymus, la valvule spirale des sélaciens, la bourse de Fabricius chez les oiseaux. Ainsi se trouve fortement conservée l'origine des cellules effectrices des réactions immunitaires.

- Origine des membres de la superfamille des immunoglobulines (Ig). La conservation des cellules engagées dans les réactions d'immunité va-t-elle entraîner une conservation des molécules effectrices? Pas nécessairement. Pour leur défense, il est évident que les animaux les plus simples ne dépendent pas uniquement de versions simplifiées des mécanismes connus chez les mammiferes [9]. Les représentants des différents embranchements du règne animal font usage selon les circonstances d'une grande variété de molécules de défense : opsonines, lectines, attacines, cécropines, dont certaines d'ailleurs sont conservées chez les vertébrés. En ce qui concerne la superfamille des immunoglobulines [5], on peut en trouver des représentants dans plusieurs embranchements, mais il semble que seuls les vertébrés les utilisent à des fins immunologiques. Les Fasciclin et Amalgam rencontrées chez plusieurs arthropodes [10] font plutôt partie de la catégorie des molécules d'adhérence cellulaire au sein de cette famille. Encore qu'Amalgam reste assez mytérieuse avec son domaine distal présentant des homologies frappantes avec les régions $\mathrm{VH}$ de vertébrés.

\section{Le système immunitaire des vertébrés, la diversité des anticorps}

Les vertébrés ne représentent qu'un seul embranchement du règne animal, en outre l'ensemble des classes de vertébrés actuels représente vraisemblablement les descendants d'une petite fraction sélectionnée des vertébrés primitifs. On peut donc s'attendre à une certaine homogénéité des composants de leur système immunitaire. En effet, tous les vertébrés étudiés jusqu'à présent rejettent les allogreffes de manière spécifique au moyen de lymphocytes d'origine mésodermique qui se sont développés dans des organes endo-mésodermiques, le plus souvent associés au tube digestif. Tous peuvent fabriquer des anticorps synthétisés par les cellules $B$, capables de reconnaître de façon spécifique un vaste spectre de déterminants antigéniques. Tous manifestent donc une certaine hétérogénéité du répertoire de leurs anticorps. Cependant, en considérant de plus près les différentes classes de vertébrés, on se rend compte de l'existence de différences dans la façon dont cette diversité est acquise ainsi que dans les degrés de cette diversité. Les deux seuls groupes vraiment distincts sont les agnathes et les gnathostomes (figure 2). Il reste beaucoup d'incertitudes quant aux capacités immunologiques des agnathes: origine et nature de leurs lymphocytes,

Figure 2. Le système immunitaire des vertébrés. Les cases rouges indiquent la présence du caractère. Les cases à moitié remplies (noires) indiquent une homologie partielle. Rec Allog : reconnaissance allogénique. $\mathrm{MHC}$ : complexe majeur d'histocompatibilité. CTL : présence de cellules $T$ cytotoxiques. $\mathrm{MHC}$ I et 11 : présence de molécules de classe I et classe II. GALT: gut associated lymphoid tissue; tissu lymphoide associé au tube digestif. NK: natural killer; cellules tueuses naturelles. T.B hétérogénéité : présence de subpopulations de lymphocytes d'origine thymique $(T)$ ou médullaire (B) capables de collaborer au cours des réponses immunitaires. MLR : réaction lymphocytaire mixte (mixed lymphocyte reaction); prolifération de lymphocytes in vitro provoquée par leur disparité au niveau des antigènes de classe II du complexe majeur d'histocompatibilité. IgM : immunoglobulines polymériques de haut poids moléculaire. $H$ : chaînes lourdes. L: chaînes légères. - : absence reconnue du caractère. (Emprunté et modifié à partir de la référence [6].) 


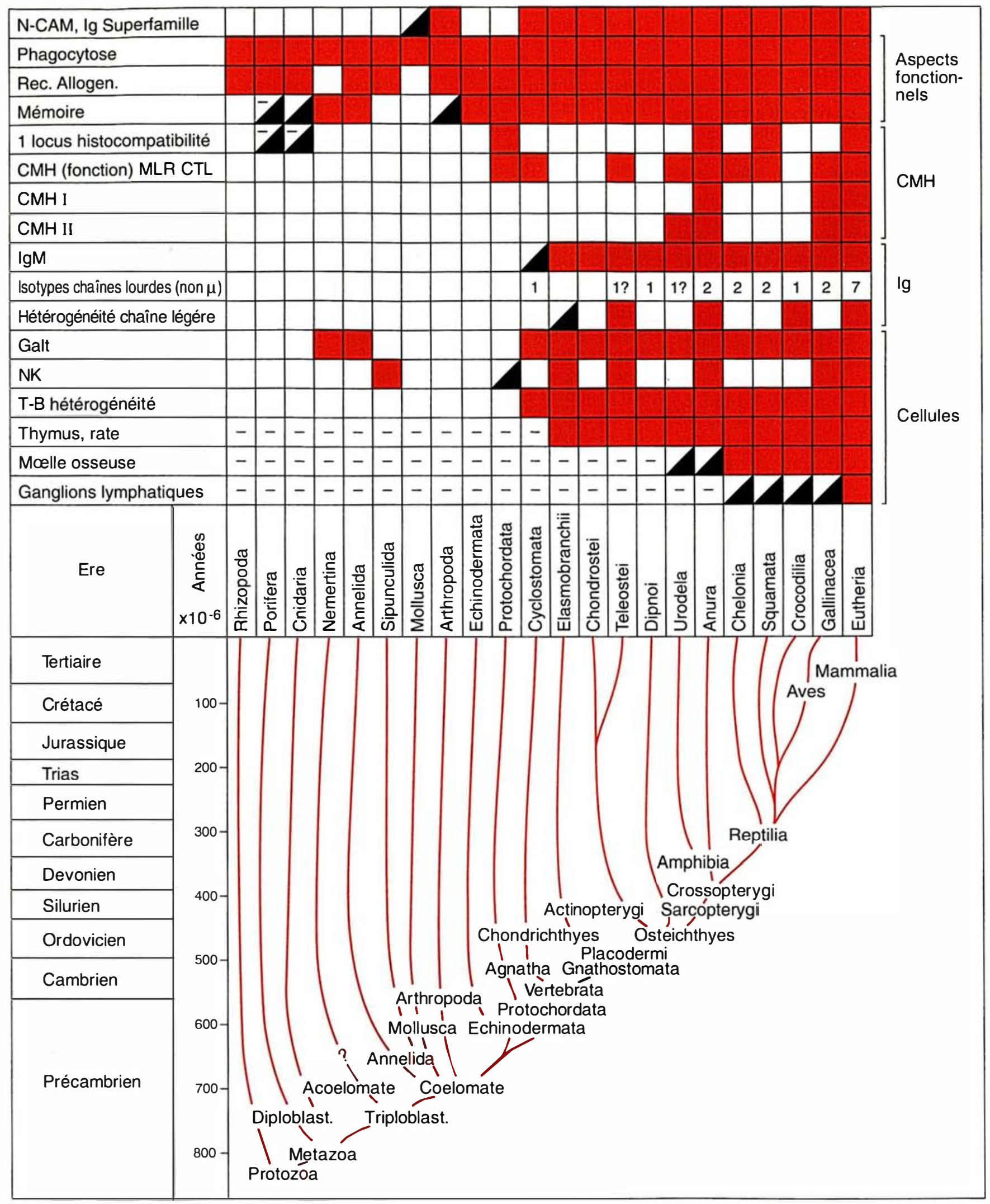


structure de leurs anticorps, présence d'un thymus, etc.

- Diversité des isotypes de chaînes lourdes et de chaînes légères. Les vertébrés primitifs ou plus exactement tous les vertébrés sauf les mammiferes possèdent un nombre restreint d'isotypes de chaînes lourdes (de 1 à 3), alors que les mammifères en possèdent jusqu'à 8 . La plupart des poissons primitifs, agnathes et chondrichthyens ainsi que les téléostéens ne possèdent que l'immunoglobuline macroglobuline polymérique de type $\operatorname{IgM}$ dont la chaîne lourde est composée de quatre domaines constants et d'un domaine variable. La filiation entre les immunoglobulines d'agnathe et les IgM classiques est incertaine puisque les deux chaînes qui sont assemblées pour former la sous-unité primordiale sont de poids moléculaire différent (revue dans [6]). Cependant, une homologie de séquence est évidente entre certains peptides de cette chaîne et les IgM humaines. La synthèse d'un deuxième isotype est possible, si elle n'est pas générale chez les poissons. La raie (chondrichthyens) a un deuxième isotype synthétisé indépendamment de l'IgM dans des cellules B spécialisées. Cette immunoglobuline est composée de deux domaines constants normaux et d'un fragment carboxy-terminal riche en cystéines et peu homologue des Ig classiques [11]. Elle possède apparemment sa propre série de gènes variables. La régulation de son expression n'est pas encore élucidée. Les amphibiens, les reptiles et les oiseaux ont généralement trois isotypes constants dont une IgM normale pentamérique et deux isotypes à quatre domaines constants qui peuvent parfois être polymériques (hexamérique dans le cas de l'IgX de Xenopus). Un de ces isotypes est généralement utilisé dans les sécrétions comme les $\operatorname{Ig} \mathrm{A}$ de mammifères, même s'il ne présente pas d'homologie structurale frappante au niveau de la séquence des gènes. C'est d'ailleurs un aspect original de l'évolution des isotypes des régions constantes. Les différents domaines les constituant semblent avoir évolué indépendamment les uns des autres pour constituer les différentes chaînes caractéristiques spécifiques des IgM ou des IgA dans un gène. Il est, en revanche, fréquent de trouver un isotype dont un domaine sera proche d'un domaine d'IgM de mammifère, mais l'autre proche d'une $\operatorname{IgG}$ de mammifère et enfin un autre plus proche d'un domaine de récepteur des cellules $\mathrm{T}$ ou d'une molécule de classe II du complexe majeur d'histocompatibilité que d'une immunoglobuline [12].

En ce qui concerne les chaînes légères, la situation est plus confuse, les vertébrés inférieurs pouvant en effet disposer d'un plus grand nombre d'isotypes que les mammifères. Le xénope possède au moins trois isotypes, comme le montrent les études au niveau des protéines et des gènes [13]. Les rapports entre ces isotypes et les chaînes $\kappa$ et $\lambda$ ne sont pas clairs et aucune filiation directe n'est à envisager. Les données suggèrent cependant que la duplication ayant donné lieu à $\mathrm{C}_{\kappa}$ et $\mathrm{C} \lambda$ a eu lieu après la séparation des lignées conduisant aux requins et aux mammifères mais avant la séparation des lignées conduisant aux amphibiens et aux mammiferes, c'est-à-dire il y a environ 400 millions d'années [14]. La plupart des études sur les chaînes légères dans les classes autres que celles des amphibiens et des sélaciens sont faites au moyen d'anticorps et de techniques d'électrophorèse ne permettant pas de déterminer les rapports entre les différents types de chaînes légères aussi bien qu'avec les données de biologie moléculaire.

- Régions variables des immunoglobulines. Organisations des gènes. En comparant les séquences des gènes des régions variables de vertébrés, on se rend compte de l'extraordinaire conservation des gènes des parties variables des immunoglobulines. Tous sont fondus dans le même moule: la protéine codée par ces gènes conservés consiste en trois régions hypervariables, ou CDR (complementarity determining region) et trois régions charpente assemblées en une succession de feuillets $\beta$ et d'hélices $\alpha$. Dans toutes les espèces, la genèse somatique d'un gène fonctionnel obéit au même mécanisme de réarrangement, entre éléments $\mathrm{V}, \mathrm{D}$ et J pour les chaînes lourdes, réarrangement entre $\mathrm{V}$ et $\mathrm{J}$ pour les chaînes légères. La structure de chaque gène, fragmenté en plusieurs éléments pourvu de signaux de recombinaisons conservés (heptamères et nonamères séparés, selon les cas, par 12 ou 23 bases), est pratiquement identique du requin à l'homme. Seuls les éléments de régulation semblent pouvoir varier (figure 3). Les différences importantes entre classes de vertébrés sont surtout visibles au niveau de l'organisation générale des gènes et par la suite de leur utilisation. Une différence majeure semble marquer la divergence chondrichthyens-ostéichthyens. Tous les chondrichthyens étudiés possèdent un assemblage linéaire en cis d'unités VDJC (au moins 200) ou VD1D2JCc au sein desquelles les réarrangements ont lieu, ainsi que des modifications somatiques du cdr3 engendrées par la fusion des $\mathrm{DH}$ ou par la diversification $\mathrm{N}$ qui consiste en l'addition au hasard de nucléotides aux extrémités des différents éléments pendant l'assemblage somatique des gènes. Dans cette espèce, on n'observe pas de réarrangements inter-unités $[15,16]$. Chez les autres vertébrés les loci d'Ig sont tous organisés selon un autre et même modèle : de nombreux éléments $\mathrm{VH}$ (chaînes lourdes) ou VL (chaînes légères) sont juxtaposés à proximité de plusieurs éléments DH (dans le cas des chaînes lourdes seulement) bordant un certain nombre de segments $\mathrm{JH}$ ou $\mathrm{JL}$ situés eux aussi à courte distance d'un gène unique de région constante. Cette configuration permet des réarrangements combinatoires qui sont une source de très grande diversité (figure 3).

- Utilisation et diversification des gènes de parties variables. Conséquences au niveau de la production d'anticorps. La différence d'organisation des gènes d'Ig entre chondrichthyens et mammifères permet d'expliquer la différence observée depuis longtemps, au niveau de la production d'anticorps, entre les individus appartenant à ces classes. Les requins ont une réponse beaucoup moins hétérogène que les souris. En effet, à nombre égal d'éléments, le requin, dépourvu de la capacité de recombiner librement tous ses éléments, produira un assortiment de parties variables beaucoup moins 


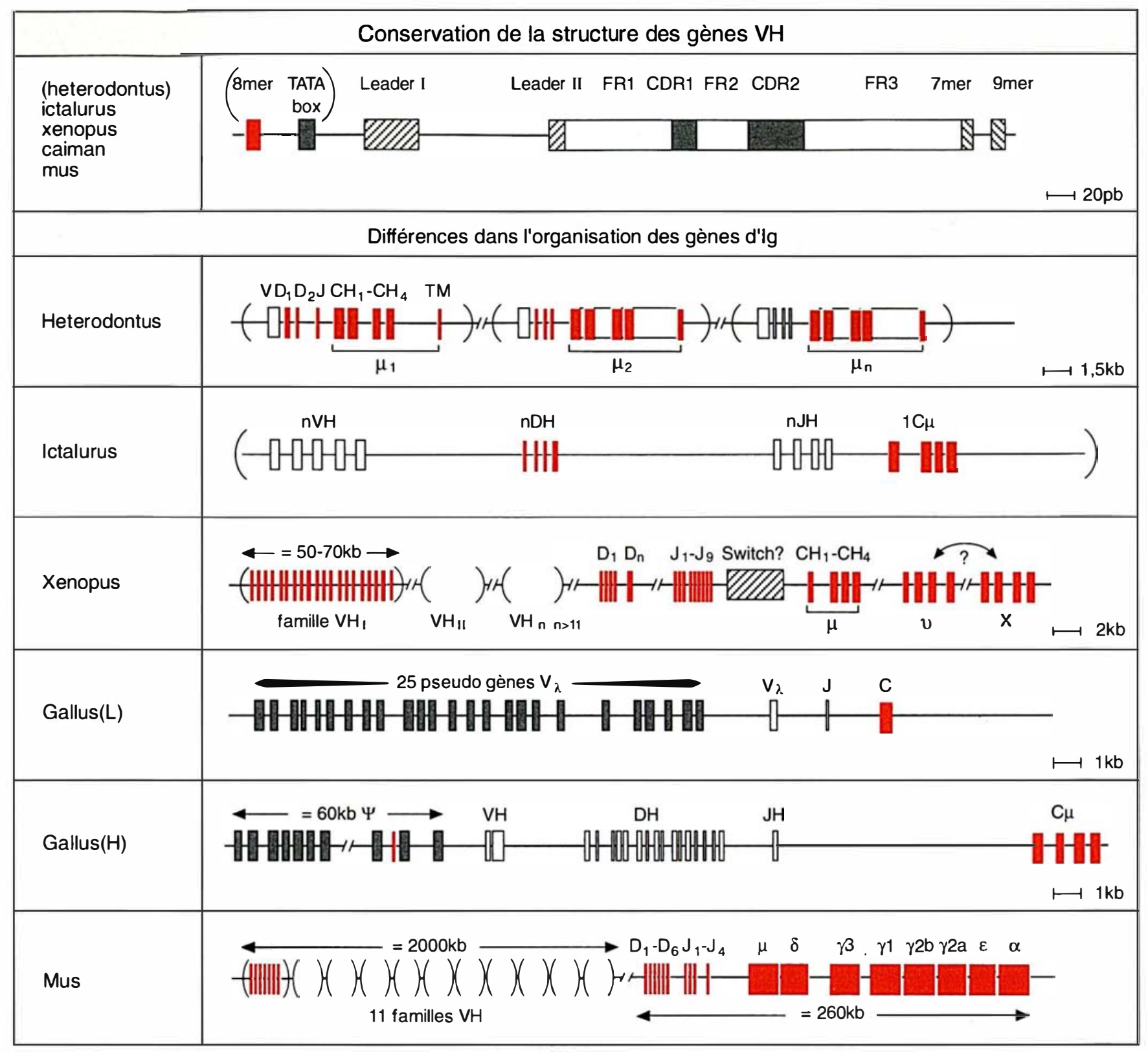

Figure 3. Les gènes d'immunoglobuline dans les différentes classes de vertébrés. Les données permettant le schéma hypothétique concernant Ictalurus sont empruntées de la référence [27]. CDR : régions hypervariables. FR; régions charpente. 7mer, 9mer: hepta- et nonamères appartenant aux signaux de recombinaison. Heterodontus : requin primitif. Ictalurus : poisson-chat. Xenopus : grenouille du Cap. Gallus : poule. Mus : souris. $\Psi$ : pseudogènes. Switch : région de commutation isotypique. L : chaîne légère; $H$ : chaîne lourde.

grand que la souris. Cela est confirmé par l'étude de la production d'anticorps chez ces animaux, qui ne montre pas de phase de maturation. Il est moins facile de comprendre la différence importante d'hétérogénéité entre les poissons osseux et les amphibiens d'une part, les vertébrés supérieurs d'autre part, puisque l'organisation de leurs gènes d'immunoglobuline est très semblable. Chez les vertébrés inférieurs, en effet, on $m / s n^{\circ} 7$, vol. 7 , septembre 91 sait depuis longtemps, grâce à des études d'isoélectrofocalisation, de réactivité croisée idiotypique et de maturation d'affinité, que l'hétérogénéité des anticorps est bien moins grande que chez les oiseaux et les mammifères. L'organisation n'explique donc pas tout. Il semble, d'une part, que la complexité du pool des gènes $V H$ puisse être moindre chez les amphibiens. Le grand nombre de familles VH [11] (revue dans [7]) ne signifie pas nécessairement une grande diversité. En raison, peutêtre, de duplications récentes ou de sélections encore mal définies, les différents éléments $\mathrm{VH}$ se ressemblent beaucoup et peuvent n'engendrer qu'une micro-hétérogénéité. D'autre part, en ce qui concerne la diversification somatique liée au réarrangement, les vertébrés inférieurs ne semblent pas limités. En revanche, l'incidence d'hypermutations somatiques 
dans les cdrl et 2 est loin d'être prouvée. Il y aurait plutôt évidence du contraire: les quelques études de biologie moléculaire dans ce domaine montrent une faible fréquence de mutations somatiques. Par ailleurs, la maturation de la réponse caractérisée par une forte augmentation d'affinité liée à la production de nouveaux variants somatiques est très faible chez les chondrichthyens, les amphibiens et les reptiles ( 10 fois au lieu de 1000 fois chez les mammifères) (revue dans [6]). Chez les oiseaux et les mammifères, les processus de diversification somatique jouent au contraire un grand rôle. Ils sont de deux types, conversion et hypermutation, et peuvent intervenir au cours de la genèse du répertoire ou au cours d'une réponse adaptative. Le processus de conversion génique dans le locus des immunoglobulines a été découvert chez les oiseaux [18]. Jusqu'à une date récente, on pensait qu'il était l'apanage des oiseaux, alors que les mammifères utiliseraient principalement les hypermutations. Il semblerait maintenant que le lapin, comme le poulet, diversifie - à partir de séquences donneuses constituées, pour la plupart, par des pscudo-gènes - l'unique réarrangement qu'il a pu effectuer [19]. L'un ou l'autre mécanisme ne sont donc pas l'apanage d'une classe de vertébrés. Le choix entre l'un et l'autre doit plutôt dépendre d'une coévolution des loci d'immunoglobulines et des modes de production de lymphocytes. En terme économique, on pourrait dire que le choix dépendra du "prix" des lymphocytes. Selon que l'individu peut ou ne peut pas gaspiller de cellules au cours de son développement. Selon la vitesse de la division cellulaire, selon que les réarrangements sont continus ou au contraire cantonnés à une période restreinte de l'ontogenèse, on peut imaginer que tel ou tel mode de diversification sera préféré [20]. Ces paramètres peuvent varier considérablement d'une classe à l'autre ou même à l'intérieur d'une espèce. Par exemple, les amphibiens deviennent immunologiquement compétents très tôt sous la double pression d'avoir à développer un système immunitaire très précocement avec un très petit
Il semble logique que l'évolution ait sélectionné chez eux un mode de diversification évitant le gaspillage en n'accordant pas de privilège aux mutations somatiques mais, plutôt, à l'expression de gènes sélectionnés de la lignée germinale. On peut même aller plus loin et risquer de prédire l'organisation de leurs gènes $V H$. En effet, si, comme dans la plupart des espèces, le réarrangement des gènes se fait en un certain ordre en commençant par les gènes $V H$ les plus proches de DH [21], il sera avantageux de rencontrer, dans ce groupe " géographiquement " privilégié, une grande variété de gènes et non pas simplement les membres souvent très semblables, sinon identiques, d'une même famille. On peut prédire que les membres des différentes familles, qui représentent des régions variables potentielles très différentes les unes des autres, seront entremêlées [22]. Pour les espèces moins "pressées ", disposant d'une longue période pour établir leur répertoire, la disposition des gènes $V H$ en groupes de membres d'une même famille n'est pas un désavantage évolutif notoire. Les espèces dont le développement est protégé du monde antigénique extérieur et dont le nombre de lymphocytes n'est pas limitant peuvent, en outre, profiter de l'avantage incontestable procuré par une diversification somatique très poussée. Il disposent ainsi d'une faculté d'adaptation individuelle bien plus grande. Une telle adaptation est la bienvenue, surtout chez des espèces dont les descendances ne sont pas très nombreuses comme les mammiferes et les oiseaux et où la valeur d'un individu est beaucoup plus grande que chez les amphibiens qui peuvent donner naissance à 2000 têtards à chaque amplexus. Au sein des espèces exploitant ce type de diversification, il semble tout d'abord qu'il y ait une corrélation entre l'absence de familles $\mathrm{VH}$ et la conversion. Le poulet possède une série de pseudo- $\mathrm{VH}$ et un $\mathrm{VH}$ fonctionnel qui, tous, appartiennent à la même famille. De même chez le lapin, où l'on semble détecter des événements de conversion, il n'existe qu'une famille VH. Il semblerait donc que l'efficacité du système de conversion soit telle que le génome puisse se débarrasser de tout un lot de gènes. On pourrait aussi avancer un argument cinétique pour expliquer la sélection de ce mode de diversification : les événements de conversion et d'hypermutation somatiques se déroulent dans le temps sur plusieurs générations cellulaires. Ils ne constitueront un outil efficace que chez les espèces produisant les cellules en grande quantité et à grande vitesse. Les oiseaux, avec un temps de division court (de l'ordre de 8 heures) et un organe spécialisé comme la bourse de Fabricius, sont bien équipés pour exploiter le potentiel des conversions [23]. Les mammifères sont dans une situation légèrement différente, puisque le répertoire précoce ne fait pas nécessairement appel aux hypermutations. Ces dernières se déroulent principalement après une immunisation et dans les centres germinatifs qui sont le siège d'une intense prolifération. Cependant, et comme preuve de la diversité des solutions, employées même au sein d'une seule classe de vertébrés, on vient de montrer que le mouton, à la différence des rongeurs, diversifie son répertoire primaire par un mécanisme d'hypermutations somatiques et de sélection dans les plaques de Peyer iléales [24]. Le système des conversions géniques laisse moins de place au hasard. Il cumule les avantages de la flexibilité somatique avec celui de la sélection de gènes utiles au cours de la phylogenèse de l'espèce. Ces derniers sont conservés sous forme de pseudogènes donneurs de séquences.

La co-évolution des loci des chaînes lourdes et des chaînes légères est remarquable. Chez les chondrichthyens, les amphibiens et les oiseaux, ces différents loci ont la même organisation génétique bien qu'ils soient situés sur deux chromosomes différents. Chez les mammifères, le locus de la chaîne $\lambda$ de la souris a une structure différente de celui des châ̂nes lourdes. Son organisation est plus proche de celle du locus contenant les gènes du récepteur des cellules $T$.

- Tendances évolutives. La comparaison des différentes solutions employées par les vertébrés pour diversifier et exprimer le répertoire de leurs immunoglobulines fait apparaître quelques tendances. Les systèmes gènes-cellules utilisant les méca- 
nismes somatiques peuvent évoluer en réduisant le nombre de leurs éléments génétiques constitutifs, alors que les systèmes essentiellement germinaux profiteront d'une multiplication compensatoire de ces mêmes éléments. Cela est une propriété des systèmes et non des espèces dans lesquelles on les rencontre. On peut en effet trouver, à l'intérieur d'une même espèce, les deux tendances représentées. Cette tendance est bien visible lorsqu'on compare le locus du récepteur des cellules $T$, connu pour son absence d'hypermutations somatiques, et le locus des immunoglobulines dans les différentes espèces. Les loci d'immunoglobulines des espèces utilisant peu ou pas les mécanismes somatiques (requins et grenouilles, par exemple) ont une structure et un mode de fonctionnement rappelant, d'une manière ou d'une autre, le locus du récepteur des cellules $\mathrm{T}$ : utilisation maximale des éléments $D$, grand nombre d'éléments J, ces deux aspects constituant une compensation à l'absence de mutations somatiques ou à leur faible incidence [22]. Le jeu de ces tendances évolutives peut être observé de façon plus vivante en étudiant les rapports entre les différents éléments du système immunitaire dans des espèces où l'on peut induire, en laboratoire, des duplications importantes par polyploïdisation. La comparaison entre ces animaux et les polyploïdes naturels, sur lesquels le temps a déjà exercé son action, nous rend compte de certaines tendances, inaccessibles autrement. Le meilleur modèle pour cette étude est l'amphibien anoure Xenopus, qui existe dans la nature sous plusieurs niveaux de ploïdie $20,36,72$, 108 chromosomes selon les espèces. La comparaison de ces polyploïdes naturels avec les polyploïdes de laboratoires révèle une tendance à conserver le plus grand nombre possible de gènes $V H$ et à se débarrasser d'un grand nombre d'allèles des gènes du complexe majeur d'histocompatibilité [25]. Les polyploïdes offrent, en outre, un intéressant modèle de contrainte évolutive, Xenopus ruwenzoriensis (108 chromosomes) est de très petite taille alors que ses cellules sont de plus grande taille que celles des espèces à 36 ou 72 chromosomes. Voici donc une espèce avec un potentiel génétique considérable qui risque d'être limité par le nombre de clones unipotentiels capables d'exprimer ces gènes. On peut imaginer que certains d'entre eux, jamais exprimés, et donc jamais sélectionnés, peuvent devenir des pseudogènes. L'existence de ces espèces polyploïdes soulève d'autres questions. L'état polyploïde a-t-il été à l'origine de l'exclusion allélique ? En effet, si on peut éventuellement imaginer que des cellules diploïdes puissent fonctionner en exprimant les deux allèles de leurs loci d'immunoglobuline, il est plus difficile d'imaginer le fonctionnement d'un système où les clones hexaploïdes exprimeraient tout leur potentiel génétique. Dans ce cas, un calcul révèle que chaque lymphocyte pourrait exprimer environ 350 variétés différentes d'immunoglobulines à sa surface, ce qui entraînerait automatiquement une moins bonne spécialisation et donc une moins bonne adaptation avec risque d'autoimmunité accru. L'exclusion isotypique et l'exclusion allélique sont donc encore plus justifiées chez les espèces polyploïdes [26].

Il faut se garder, lorsqu'on étudic l'évolution du système immunitaire, de conclure trop vite au caractère primitif d'un système. En raison de l'effet " autocatalytique " des duplications, les loci d'immunoglobuline peuvent sans doute évoluer beaucoup plus rapidement que les autres loci d'une espèce. En guise d'exemple, au sein d'une même classe de vertébrés comme les amphibiens et pour un niveau de ploïdie donné, il n'cst pas rare de trouver des différenc's très importantes dans la multiplicité des gènes du système immunitaire. Il est donc difficile de rencontrer un système immunitaire primitif chez les vertébrés actuels. Il est vraisemblable que toutes les organisations génétiques rencontrées sont toutes le produit d'une évolution très avancée, peutêtre récente, fruit de leur adaptation aux conditions de production et à l'économie de leurs cellules lymphoïdes. Chez le xénope il est peut-être possible de suivre cette évolution en action. En effet, chez cet animal, l'instabilité semble être liée à la présence de transposons ou de structures ressemblant à des transposons qui pourraient favoriser les échanges entre segments du génome. Ainsi, d'un allèle à l'autre du même gène, on peut rencontrer un polymorphisme délimité par ces structures. Dans le cas du locus DH de cette espèce, on a même pu assister à la genèse d'un tel polymorphisme à l'intérieur de clones d'animaux en l'espace d'une quinzaine d'années seulement.

En conclusion, l'étude de l'évolution du système immunitaire, et de la diversité des anticorps en particulier, nous révèle un monde particulièrement dynamique et varié. La comparaison des solutions choisies par les différentes espèces devrait nous permettre de mieux distinguer l'essentiel de l'accessoire dans l'étonnante multiplicité des éléments qui entrent en jeu. De ce point de vue, l'approche phylogénique n'a pas seulement un intérêt fondamental, mais aussi un intérêt appliqué qui pourra éventuellement suggérer des solutions aux praticiens lorsqu'ils auront à faire face à des problèmes de carences du système immunitaire chez l'homme

\section{Summary}

Evolution of the immune system : the origin of antibody diversity

The immune system of vertebrates started to develop very carly in evolution, about 600 million years ago when appeared in primitive fish most of the genes and cells specific to this system. The solutions chosen during phylogeny in order to generate antibody and $\mathrm{T}$ ccll receptor repertoires represent variations on a theme, calling in a variable manner combinatorial rearrangement of genctic elements, gene conversion and somatic mutations and hypermutations. The selected systems reflect more a good adaptation to features characteristic of the cellular dynamics of the immune system than the filiation relationships between the species : duration of the period during which the repertoire is produced, number of lymphocytes available, possibility of wastagc, mitotic activity of cells, etc.

\section{TIRÉS A PART}

L. Du Pasquier. 